\title{
Modificación de los parámetros metabólicos y microalbuminuria en pacientes con diabetes tipo 2 tratados con acarbosa
}

\author{
E. GONZÁLEZ SARMIENTO, P. ERGUETA MARTÍN, I. FERNÁNDEZ MARTÍNEZ, \\ M.C. HINOJOSA MENA-BERNAL, I. ZURRO MUÑOZ, J. ZURRO HERNÁNDEZ
}

Departamentos de Medicina Interna y Endocrinología. Hospital Universitario. Valladolid

\author{
MODIFICATION OF THE METABOLIC PARAMETERS AND MICRO - \\ ALBUMINURIA IN PATIENTS WITH DIABETES MELLITUS TYPE 2 \\ TREATED WITH ACARBOSE
}

\section{RESUMEN}

Objetivo: Determinar la influencia de la acarbosa en la microalbuminuria y parámetros metabólicos en pacientes con diabetes mellitus tipo 2.

Material y métodos: Hemos estudiado 92 pacientes con diabetes tipo 2 , tratados con acarbosa, sola o con insulina o antidiabéticos orales. Hemos determinado los valores de $\mathrm{Hb} \mathrm{A} 1 \mathrm{c}$, colesterol total, colesterol HDL, colesterol LDL, triglicéridos y microalbuminuria, antes y después del tratamiento con acarbosa

Resultados: Los pacientes presentaron de forma global una mejoría estadísticamente significativa de los niveles de HbAlc, triglicéridos y microalbuminuria. En el grupo tratado con $30 \mathrm{mg}$ de acarbosa al día se observó un descenso significativo de los valores de Hba1c y triglicéridos, independientemente del tratamiento asociado. En el grupo tratado con $150 \mathrm{mg}$ de acarbosa al día la mejoría fue estadísticamente significativa para los niveles de HbA1c.

Conclusiones: El tratamiento con acarbosa produce una mejoría en los niveles de $\mathrm{HbA} 1 \mathrm{c}$, independientemente de la dosis administrada, y de los niveles de triglicéridos en pacientes tratados con $300 \mathrm{mg}$ de acarbosa al día, sin relación con los tratamientos asociados. Por esto, la acarbosa debe ser considerada como una medicación efectiva para mejorar el control metabólico en vistas a prevenir el riesgo cardiovascular en estos pacientes.

\begin{abstract}
Objective: to determinate the influence of the acarbosa and microal buminuria on metabolic parameters in patients diabetics type 2.

Research desing and methods: We are studied 92 patients with dia betes type 2, treated with Acarbose alone or with insuline or oral anti diabetics We are determinated the values of HbAlc, total cholesterol, $H D L, L D L$, triglycerides and microalbuminuria, before and after or the treatment with acarbose.

Results: The patients presented globally a statistically significant improvement in the levels of $\mathrm{Hb} A 1 \mathrm{c}$, triglycerides and microalbuminu ria. In the group treatise with $300 \mathrm{mg} /$ day of acarbose it was observed a significant decrease of the values of $\mathrm{Hb} A \mathrm{Alc}$ and triglycerides indepen dently of the associated treatment. In the group treated with $150 \mathrm{mg} /$ day the improvement only it was statistically significant for the levels of $\mathrm{Hb}$ Alc.

Conclusions: The treatment with acarbose produces an improvement in the levels of $\mathrm{Hb} \mathrm{Alc}$ independently of the administered dose and of the triglycerides in patients treated wth $300 \mathrm{mg} /$ day without relation to associated treatments Therefore, he acarbose win be considered an effective medication to improve the metabolic contral to prevent the cardiovascu lar risk in those patients
\end{abstract}

KEY WORDS: Acarbose. Lipids. Microalbuminuria.

González Sarmiento E, Ergueta Martín P, Fernández Martínez I, Hinojosa Mena-Bernal MC, Zurro Muñoz I, Zurro Hernández J. Modifica ción de los parámetros metabólicos y microalbuminuria en pacientes con diabetes tipo 2 tratados con acarbosa. An Med Interna (Madrid) 2001; 18: 234-236.

\section{INTRODUCCIÓN}

La acarbosa es un inhibidor de la alfa glucosidasa intestinal que actúa inhibiendo de forma competitiva la absorción de carbohidratos, Reduce la hiperglucemia postprandial y la hiperinsulinemia (1), lo cual conduce a un descenso moderado de los niveles de hemoblogina glicosilada $(2,3)$.

Las indicaciones fundamentales de la acarbosa son el tratamiento de pacientes diabéticos tipo 2 con obesidad, bien sola o asociada con antidiabéticos orales o insulina, la hiperglucemia postprandial y la hiperinsulinemia (4).
Durante la fase postprandial, la pared vascular en los pacientes diabéticos es sometida a elevadas concentraciones de glucosa y triglicéridos que dañan el endotelio y estimulan el desarrollo de complicaciones macro y microvasculares (5). Debido a esto, algunos autores han relacionado la hiperglucemia e hiperinsulinemia con un incremento de la morbimortalidad por cardiopatía isquémica $(6,7)$, que es altamente prevalente en caso de nefropatía y retinopatía diabética (8). La hiperglucemia postprandial se ha relacionado también con los niveles de microalbuminuria, siendo considerada hoy como un marcador de riesgo cardiovascular $(9,10)$.

Trabajo aceptado: 20 de Diciembre de 2000 
La incidencia de efectos secundarios gastrointestinales fue inferior cuando la dosis se administró de forma progresiva, y a mayor duración del tratamiento. Se ha demostrado una buena tolerancia en pacientes diabéticos tipo 2 , sobre todo en los tratados con dosis de $150 \mathrm{mg}$ al día en los tipo 1 (11).

El objetivo de este estudio es valorar el efecto del tratamiento con acarbosa sobre la microalbuminuria, la $\mathrm{HbA} 1 \mathrm{c}$, colesterol total, colesterol HDL, colesterol LDL y triglicéridos en pacientes con diabetes mellitus tipo 2 .

\section{MATERIAL Y MÉTODOS}

Se recopilaron las historias clínicas de 92 pacientes diabéticos tipo 2 (según los criterios de la OMS) procedentes del Departamento de Medicina Interna y Endocrinología del Hospital Universitario de Valladolid. 46 fueron varones (50\%) y 46 mujeres (50\%), con una edad media de 61,22 $\pm 11,83$ años, siendo la edad mínima 36 y la máxima 87 años; y con un índice de masa corporal (BMI) superior a $27 \mathrm{Kg} / \mathrm{m}^{2}$.

Los pacientes recibieron tratamiento con acarbosa a dosis de $150 \mathrm{mg} /$ día o $300 \mathrm{mg} /$ día, bien sola o asociada con insulina o sulfonilureas.

La dosis de acarbosa se estableció de acuerdo al grado de compensación metabólica, valorada según el perfil glucémico y los niveles de HbA1c, y el grado de tolerancia digestiva. La dosificación se administró de forma progresiva, incrementándose en $50 \mathrm{mg}$ semanales hasta completar la dosis total, dividida en tres tomas diaria, e ingiriéndola junto con el primer bocado de cada comida principal.

53 de los 92 pacientes $(57,60 \%)$ recibieron una dosis total de $300 \mathrm{mg} /$ día de acarbosa, y 39 pacientes $(42,39 \%)$ de 150 $\mathrm{mg} /$ día.

De los 53 pacientes tratados con dosis de $300 \mathrm{mg} /$ día de acarbosa, 26 (49\%) eran mujeres y $27(51 \%)$ varones, con una edad media de 56,58 $\pm 10,94$ (rango entre 41 y 79 años); 5 $(9,43 \%)$ en tratamiento con insulina, $20(37,73 \%)$ con sulfonilureas, y $28(52,83 \%)$ sólo con acarbosa.

De los 39 pacientes tratados con dosis de acarbosa de 150 gm/día, 21 (54\%) eran mujeres y 18 (46\%) varones, con una edad media de $62,06 \pm 12,30$ (rango entre 36 y 87 años); 2 $(5,12 \%)$ con insulina, $11(28,20 \%)$ con sulfonilureas y 26 $(66,66 \%)$ sólo con acarbosa (Tabla I).

De los 92 pacientes iniciales todos completaron el estudio, no presentándose ningún caso de efectos secundarios gastrointestinales y otro tipo de efectos que condujeran al abandono del tratamiento.

Mediante autoanalisis se determinaron, al comienzo y a los 6 meses de tratamiento, los parámetros hematológicos de

\section{TABLA I}

\section{CARACTERÍSTICAS EPIDEM IO LÓ GICAS DE LOS PACIENTES}

\begin{tabular}{lcc}
\hline Dosis de Acarbosa & $300 \mathrm{mg} / \mathrm{día}$ & $150 \mathrm{mg} / \mathrm{día}$ \\
No de pacientes & $53(57,6 \%)$ & $39(42,39 \%)$ \\
M ujeres & $260(49 \%)$ & $21(54 \%)$ \\
Varones & $27(51 \%)$ & $18(46 \%)$ \\
Edad (años) & $56,58 \pm 10,94$ & $62,06 \pm 12,30$ \\
Insulina + Acarbosa & $5(9,43 \%)$ & $2(5,12 \%)$ \\
Sulfonilureas + Acarbosa & $20(37,73 \%)$ & $11(28,20 \%)$ \\
Acarbosa sola & $28(52,83 \%)$ & $26(66,66 \%)$ \\
\hline
\end{tabular}

rutina y tests bioquímicos. La glucosa plasmática se determinó por el método de oxidación de la glucosa, la HbA1c mediante cromatografía in columna (valores estándar: 4,7-6,4\%), la microalbuminuria por nefelometría, y el colesterol total (CT), triglicéridos (TG) y HDL por métodos enzimáticos de espectrofotometría. Los niveles de LDL colesterol se calcularon usando la fórmula de Friedewald: $\mathrm{LDL}=\mathrm{CT}-$ HDL - (TG/5).

El estudio estadístico se realizó mediante: a) contraste de hipótesis de datos apareados basado en la distribución normal, considerando valores significativos aquellos con $p<0,05$; b) contraste de la diferencia de medias de dos poblaciones normales independientes, basado en la distribución de la t de Student para la comparación entre la población con dosis de 150 $\mathrm{mg} /$ día y la de $300 \mathrm{mg} /$ día. Los resultados se expresan como la media \pm la desviación estándar.

\section{RESULTADOS}

Los 92 pacientes estudiados completaron los 6 meses de tratamiento con acarbosa, por lo que todos fueron incluidos en el estudio estadístico.

En las tablas II, III y IV se expresan los valores obtenidos en condiciones basales y tras la administración de la medicación, con su significación estadística $(\mathrm{p}<0,05)$.

En el grupo de pacientes tratado con $300 \mathrm{mg} /$ día de acarbosa, e independientemente del tratamiento asociado, se observó un descenso en los niveles de HbA1c $(\mathrm{p}<0,001)$ y de triglicéridos ( $\mathrm{p}<0,01)$. En el grupo tratado con $150 \mathrm{mg} /$ día de acarbosa, la significación estadística sólo se reflejó en el caso de los valores de HbA1c $(\mathrm{p}<0,05)$.

\section{TABLA II}

M ODIFICACIÓN DE LOS PARÁM ETROS EN EL GLOBAL DE PACIENTES

\begin{tabular}{lccc}
\hline & Comienzo & A los 6 meses & \\
\hline HbA1c (\%) & $7,10 \pm 1,69$ & $6,44 \pm 1,60$ & $p<0,001$ \\
Colesterol (mmol/l) & $5,83 \pm 1,13$ & $5,73 \pm 1,11$ & NS \\
C-HDL (mmol/I) & $1,39 \pm 0,47$ & $1,27 \pm 0,50$ & NS \\
C-LDL (mmol/l) & $3,96 \pm 1,03$ & $3,96 \pm 0,98$ & NS \\
Triglicéridos (mmol/I) & $1,87 \pm 1,20$ & $1,50 \pm 0,71$ & $p<0,05$ \\
M icroalbuminuria & & & \\
$\quad$ (mg/24 h) & $52,04 \pm 76,52$ & $31,55 \pm 51,46$ & $p<0,05$ \\
\hline
\end{tabular}

TABLA III

MODIFICACIÓ N DE PARÁM ETROS EN EL GRUPO TRATADO CON 150 M G/DÍA DE ACARBOSA

\begin{tabular}{lccc}
\hline & Comienzo & A los 6 meses & \\
\hline HbA1c (\%) & $6,98 \pm 1,91$ & $6,44 \pm 1,67$ & $\mathrm{p}<0,05$ \\
Colesterol (mmol/l) & $5,70 \pm 1,02$ & $5,51 \pm 5,05$ & $\mathrm{NS}$ \\
C-HDL (mmol/l) & $1,39 \pm 0,41$ & $1,45 \pm 0,50$ & $\mathrm{NS}$ \\
C-LDL (mmol/l) & $3,69 \pm 1,01$ & $3,69 \pm 1,16$ & $\mathrm{NS}$ \\
Triglicéridos (mmol/l) & $2,30 \pm 1,68$ & $1,69 \pm 0,76$ & $\mathrm{NS}$ \\
M icroalbuminuria & & & \\
$\quad$ (mg/24h) & $54,98 \pm 75,97$ & $21,76 \pm 28,83$ & $\mathrm{NS}$ \\
\hline
\end{tabular}


TABLA IV

M ODIFICACIÓN DE PARÁM ETROS EN EL GRUPO TRATADO CON 300 M G/DÍA DE ACARBOSA

\section{Comienzo A los 6 meses}

$\begin{array}{lccc}\text { HbAlc (\%) } & 7,11 \pm 1,42 & 6,37 \pm 1,51 & \mathrm{p}<0,001 \\ \text { Colesterol (mmol/l) } & 5,81 \pm 1,21 & 5,74 \pm 1,08 & \text { NS } \\ \text { C-HDL (mmol/l) } & 1,31 \pm 0,45 & 1,20 \pm 0,50 & \text { NS } \\ \text { C-LDL (mmol/l) } & 4,14 \pm 1,04 & 4,14 \pm 1,92 & \text { NS } \\ \text { Triglicéridos (mmol/l) } & 1,41 \pm 0,49 & 1,18 \pm 0,34 & p<0,01\end{array}$

Microalbuminuria

(mg/24 h) $\quad 23,40 \pm 39,0918,14 \pm 25,08 \quad$ NS

\section{DISCUSIÓN}

El tratamiento con acarbosa en diabéticos tipo 2, solo o asociado con sulfonilureas o insulina, durante un periodo de 6 meses, conduce a una mejoría significativa en el control glucémico, medido mediante los niveles plasmáticos de $\mathrm{HbA} 1 \mathrm{c}$, independientemente de la dosis de acarbosa utilizada (150 ó $300 \mathrm{mg} /$ día), de acuerdo con lo observado por la mayoría de autores $(13,14)$.

En el grupo de pacientes tratados con $300 \mathrm{mg} /$ día de acarbosa encontramos un descenso significativo de los niveles de triglicéridos plasmáticos, que podría estar en relación con el mejor control glucémico. Este hecho no se apreció en pacien- tes tratados con dosis de acarbosa de $150 \mathrm{mg} /$ día, a pesar de la mejoría en los niveles de glucemia, y puede representar un efecto beneficioso del tratamiento con acarbosa dosis dependiente.

La acción de la acarbosa sobre los lípidos plasmáticos está sometida a controversia, siendo confirmada por Hanefeld (1) sobre los triglicéridos, y por Austenat (15) sobre el colesterol, pero en diabéticos tipo 1 .

Dado que actualmente la microalbuminuria es considerada como un marcador de riesgo cardiovascular, y que en diabéticos tipo 2 depende, entre otros factores, de los niveles de glucemia, hemos evaluado este parámetro, encontrando un descenso del mismo en todos los grupos de tratamiento, lo cual es solamente significativo considerando los pacientes de una forma global, e independientemente de la dosis administrada.

Este descenso de la microalbuminuria no se ha demostrado hasta la fecha, salvo en estudios experimentales (16). Nosotros pensamos que pudiera tener gran trascendencia dada la alta prevalencia de eventos cardiovasculares presentes en estos pacientes. En orden a confirmar este hallazgo serían precisos largas series de estudios ya que cuando el grupo global de pacientes es fraccionado la muestra podría ser insuficiente.

En resumen, el tratamiento con acarbosa en pacientes con diabetes mellitus tipo 2 mejora los niveles de $\mathrm{HbA1c}$, independientemente de la dosis administrada (150 ó $300 \mathrm{mg} /$ día); y de triglicéridos a dosis de $300 \mathrm{mg} /$ día. Es posible que esta dosis tenga un efecto beneficioso sobre la microalbuminuria. La acarbosa puede ser considerada como una medicación efectiva en estos pacientes, con la intención de mejorar su control metabólico y prevenir el riesgo cardiovascular, tan elevado en ellos.

\section{Bibliografía}

1. Hanefeld M, Fisher S, Schulze J, Spengler M et al. Therepeutic potentials of acarbose as first line drug in NIDDM insufficienly treated with diet alone. Diabetes Care 1991; 14: 732-737.

2. Balfour JA, McTavish D. The beneficial effects of acarbose on postprandial glucose levels have been confirmed in patients with non-insulin-dependent diabetes mellitus and postprandial insulin and triglyceride levels were also lowered. Drugs 46 (1993): 1025-1054.

3. Chiasson JL, Losse RG, Hunt JA, Palmason C et al. The efficacy of acarbose en treatment of patients with non-insulin-dependent diabetes mellitus. A multicenter controlled clinical trial. Ann Intern Med 121 (1994): 928-935.

4. Menhert H. Diabetes mellitus und orale Antidiabetika. Akt Pharmakol 1994; 33: 293.

5. Haller H, Drab M, Luft FC. The role of hyperglycemia and hyperinsulinemia en the pathogenesis of diabetic angiopathy. Clinical Nephrology 1996; 46: 246-255.

6. Hanefeld M, Fisher S, Julius U, Schulze J et al. Risk factors for myocardial infartion and death in newly detected NIDDM: The diabetes Intervention Study, 11 year-follow-up. Diabetología 1996; 39: 1577-1583.

7. Donahue RP, Abott RD, Reed DM, Yano K. Postchallenge glucose concentration and coronary heart disease en men of Japanese ancestry: Honolulul Heart Program. Diabetes 1987; 36: 689-692.

8. Stolk RP, Vigerling JR, Jong P TVM, Dielemans I et al. Retinopathy,

glucose, and insulin en an elderly population. The Rotterdam Study. Diabetes 1995; 44: 11-15.

9. Parving HH, Hommel E. Prognosis in diabetes nephropathy. BMJ 1989; 299: 230-233.

10. Izzo JL JR. Primer de hipertension. (American Heart Association) 1996

11. Zurro Hernández J, Ergueta Martín P, Fernández Martínez I, Hinojosa Mena-Bernal MC et al. Estudio de la tolerancia digestiva en 202 pacientes con DM tipo 2. Av Diabetol. 1997; 13: 116-119.

12. Martí Colomer J, Tur Cotaina MD, Martín Baena J. Acarbosa en el control metabólico de la diabetes mellitus infantol juvenil. Resultados a medio plazo. An Med Interna (Madrid) 1995; 12: 382-387.

13. Josse RG. Acarbose for the treatment of tipe II diabetes: The result of a Canadian multicenter trial. Diabetes research and Clinical Practice 28 Suppl. (1995) S 167- S 172.

14. Costa B, Piñol C. Acarbose in ambulatory treatment of non-insulindependent diabetes mellitus associated to inminent sulfonylurea failure: A randomised multicenter trial in primary health care. Diab Res Clin Prac 38, 1997; 33-40.

15. Austenat E. Recent therapy studies with acarbose in type I diabetics. Endokrinologie und Stoffwechsel 1991; 12: 19-24.

16. Cohen MP, Vasselli JR, Neumn RG, Witt J et al. Treatment with acarbose, an alpha glucosidase inhibitor, reduces increased albumin excretion in streptozotocin diabetic rats. Gen Pharmacol 1995; Oct. 26 (6), 1355-1361. 